\title{
Nonlinear singular perturbation problems
}

\section{John James Shepherd}

This thesis examines singular perturbation problems $P_{\varepsilon}$ that involve second order ordinary differential operators on a finite interval. Specifically, it is assumed that $P_{\varepsilon}$ takes the form

$$
\begin{aligned}
\varepsilon y^{\prime \prime}+f\left(x, y, y^{\prime}, \varepsilon\right) & =0,0<x<1, \\
a_{1}(\varepsilon) y^{\prime}(0, \varepsilon)+b_{1}(\varepsilon) y(0, \varepsilon) & =A(\varepsilon), \\
a_{2}(\varepsilon) y^{\prime}(1, \varepsilon)+b_{2}(\varepsilon) y(1, \varepsilon) & =B(\varepsilon),
\end{aligned}
$$

where $a_{i}, b_{i}, A, B$, and $f$ are known functions, and $\varepsilon$ is a small real positive parameter. Such problems have relevance in many branches of the physical sciences, and have received considerable attention over recent years. Three basic questions are pertinent:

(a) does the given problem have a solution;

(b) is this solution unique in any sense; and

(c) what are the asymptotic properties, as $\varepsilon \rightarrow 0$, of this solution?

These questions have been answered to some degree by a number of writers. Most have presupposed the existence of an "approximate solution" $w(x, \varepsilon)$ that satisfies $P_{\varepsilon}$ "approximately" in some suitably defined sense. It is then shown that $P_{\varepsilon}$ has an exact solution $y(x, \varepsilon)$ in an appropriately small neighbourhood of $w(x, \varepsilon)$. The construction of the function $w(x, \varepsilon)$ may be carried out by heuristic techniques; although this aspect is often neglected entirely, being seen as a separate issue.

Received 3 June 1977. Thesis submitted to the University of Melbourne, January 1977. Degree approved, May 1977. Supervisor: Professor Simon Rosenblat. 
This thesis differs in that it is proposed that $w(x, \varepsilon)$ be the solution of a suitably chosen linear problem $L_{\varepsilon}$. For such a problem, the questions $(a),(b)$, and $(c)$ are readily answered. We then aim to establish an equivalence between the problems $P_{E}$ and $L_{\varepsilon}$, for small $\varepsilon$, to facilitate the comparison of $y(x, \varepsilon)$ and $w(x, \varepsilon)$.

The procedure is a standard one. We set

$$
y(x, \varepsilon)=w(x, \varepsilon)+u(x, \varepsilon)
$$

and linearize about $w(x, \varepsilon)$, obtaining a problem for $u(x, \varepsilon)$; which is then converted to an integral equation format by the construction of the appropriate Green's function. The existence of a solution $u(x, \varepsilon)$ that is small in some sense is then established by use of the contraction mapping theorem.

Chapter II deals with linear problems, and the equivalence criteria established are used to validate the approximate solutions obtained by heuristic techniques.

Chapter III deals with nonlinear problems. For the semilinear case, where $f$ is linear in $y^{\prime}$, it is shown that the existence of a suitable function $w$, satisfying the problem $L_{\varepsilon}$, depends entirely on the existence of functions $w_{0}$ and $w_{1}$ that satisfy equations obtained by heuristic means. This gives a justification of the so-called "matching techniques".

Chapters IV and $V$ deal with specific nonlinear examples that have some physical interest. In Chapter IV, we examine the so-called Reynolds equation, modelling the flow of gas in a gas bearing at high bearing numbers. In Chapter $V$, a problem having relevance to chemical reactor theory is dealt with, and a technique for constructing the problem $L_{\varepsilon}$ is investigated. In both cases, answers to (a), (b), and (c) are obtained, under suitable hypotheses. 